\title{
Morphometric study of the bilateral middle cerebral arteries in patients with a unilateral aneurysm
}

\author{
Zygmunt Siedlecki $^{1} \oplus$, Karol Nowak ${ }^{1} \oplus$, Maciej Śniegocki ${ }^{1} \oplus$, \\ Magdalena Grzonkowska², Mariusz Baumgart² ${ }^{\oplus}$, Marcin \\ Wiśniewski² ${ }^{\circ}$, Michał Szpinda² $₫$
}

\begin{abstract}
${ }^{1}$ Department of Neurosurgery and Neurotraumatology and Pediatric Neurosurgery, Collegium Medicum in Bydgoszcz of Nicolaus Copernicus University in Toruń, Poland

${ }^{2}$ Department of Anatomy, Collegium Medicum in Bydgoszcz of Nicolaus Copernicus University in Toruń, Poland
\end{abstract}

\begin{abstract}
Background: The middle cerebral artery (MCA) supplies a major part of the brain and is of considerable clinical importance as a common location of intracranial aneurysms. The aim of the present study was to analyze the bilateral MCAs in patients with unilateral aneurysms. Material and methods: Images of computed tomography angiography of 45 patients were analyzed. The morphometric parameters of MCA were measured. The measured parameters of the normal MCAs were compared with MCAs containing aneurysms located in the main trunk division. Results: No significant differences in the length of trunk and angles between the middle and anterior cerebral arteries were found between the non-affected and aneurysmatic MCAs. Concerning arteries with aneurysms, more branches originating from the main trunk division were found more frequently. The coexistence of the more frequent trifurcation of the MCA and an aneurysm constitutes evidence of greater anatomical variability of this arterial area in cases with a concomitant vascular malformation. Conclusions: This finding is consistent with literature data, showing the relationship between aneurysms and arterial variability and the fact that aneurysms are most often located at arterial divisions.
\end{abstract}

Keywords: computed tomography angiography $\cdot$ intracranial aneurysm $\cdot$ middle cerebral artery $\cdot$ morphometry

\section{Citation}

Siedlecki Z, Nowak K, Sniegocki M, Grzonkowska M, Baumgart M, Wiśniewski M, Szpinda M. Morphometric study of the bilateral middle cerebral arteries in patients with a unilateral aneurysm. Eur J Transl Clin Med. 2021;4(1):29-34. DOI: $10.31373 /$ ejtcm/130440

Funding: Neurosurgery Department and the Department of Normal Anatomy of the Ludwik Rydygier Collegium Medicum in Bydgoszcz Available online: www.eitcm.gumed.edu.pl 


\section{Abbreviations}

- ACA - anterior cerebral artery

- CT - computed tomography

- CTA - computed tomography angiography

- DICOM - digital imaging and communications in medicine

- DSA - digital subtraction angiography

- ICA - internal carotid artery

- MCA - middle cerebral artery

- MRA - magnetic resonance angiography

- $\mathrm{MRI}$ - magnetic resonance imaging

- SAH - subarachnoid hemorrhage

\section{Introduction}

The middle cerebral artery (MCA) is the largest terminal branch of the internal carotid artery (ICA), which delivers blood to most of the cerebral hemisphere [1-2]. Circulatory disturbances inside the MCA cause ischemic strokes with focal neurological deficits [3]. The central branches of the MCA play an essential role in brain hemorrhage because they may often rupture due to atherosclerotic lesions or arterial hypertension [2]. The MCA is also a location of saccular cerebral aneurysms [3-4]. Aneurysms of the MCA are most often located within its main trunk division where hemodynamic stress is greatest [4-5].

From an anatomical point of view, the MCA is a direct extension of the ICA, with the anterior cerebral artery (ACA) being the second major branch originating from the ICA. The MCA is separated into five segments: M1 - M5. The first segment (M1, also known as the sphenoidal segment) is positioned horizontally and passes in the lateral direction. Subsequently, at the limen insulae the M1 enters the lateral sulcus of the brain, where it splits into M2 branches [1-2]. Most frequently, such a division is bifurcated, but there exists some variability with more than two M2 branches [6-7]. Since the $\mathrm{M} 2$ branches extend in the posteriorly within the lateral sulcus and surround the insula, the $M 2$ is named the insular segment $[2,6]$. In the region of the operculae, the M2 branches further split into the M3 branches, also called the opercular segment. In the region of the three operculae covering the lateral sulcus, the M3 branches extend to the cerebral convexity and are distinguished as the M4 cortical segments, which eventually split into the terminal (M5) segments [2].

MCA divisions are the third most frequent location of saccular cerebral aneurysms. MCA aneurysms constitute approximately $20 \%$ of all intracranial aneurysms [8]. Saccular cerebral aneurysms occur in approximately $2 \%$ of the entire population [8-9]. Cerebral vessels with potential vascular malformations may be visualized using three basic diagnostic methods: digital subtraction angiography (DSA), computed tomography angiography (CTA) and magnetic resonance angiography (MRA) $[7,10]$. CTA used in this article is commonly considered in clinical practice. It is a rapid and accessible imaging test which can be performed at the Emergency Departments. CTA allows an immediate detection of cerebral aneurysms [10]. The aim of this study was a CTA-based comparative analysis of the bilateral MCAs in patients with unilateral saccular aneurysms localized within the M1 division.

\section{Material and methods}

We performed a retrospective morphometric analysis of CTA images obtained from 45 patients who were treated at a single neurosurgery center in the in the years 2018-2019. The reason for referral was unilateral intracranial aneurysm located in the M1 division of the MCA.

CTAs in patients with unruptured aneurysms were performed in an outpatient setting. The CTA scans were acquired $0.4 \mathrm{~mm}$ intervals using a Discovery CT $750 \mathrm{HD}$ (General Electric Healthcare, Chicago, USA) scanner and were saved in the Digital Imaging and Communications in Medicine (DICOM) format. To visualize their blood vessels, patients were administered $80 \mathrm{ml}$ of intravenous contrast agent (Omnipaque by General Electric Healthcare, Chicago, USA). The exclusion criteria were: comorbidities that could affect the cerebral vessel morphology (e.g. polycystic kidney disease), coexisting cerebral aneurysm at a different location, an expansive intracerebral hematoma in the area of the MCA aneurysm, the M1 trunk not visible in CTA due to massive cerebral edema. In all patients with subarachnoid hemorrhage (SAH), a computed tomography (CT) examination was performed to diagnose $\mathrm{SAH}$, and immediately afterwards a CTA examination was done. $\mathrm{SAH}$ visualized in these CTAs was considered acute, therefore cerebral vasospasm was not taken into account in the measurement of the MCA width.

The analysis (comparisons of MCAs with M1 division aneurysm and non-affected MCAs) was performed by two authors: a neurosurgery specialist with 15 years of professional experience in aneurysm surgery and an anatomist with 10 years of professional experience in morphometry.

The following five parameters were measured:

\section{1. length of M1 measured bilaterally from the ICA division to the $M 1$ bifurcation,}

2. width of $M 1$ in the proximal section of the artery,

3. width of $\mathrm{M} 1$ in the middle section of the artery, 
4. width of $M 1$ next to the bifurcation and

5. the angle between $\mathrm{M} 1$ and the ACA A1 segment measured in the plane formed by the proximal branches of these two arteries (the angle at which the terminal branches of the ICA bifurcate).

Statistical analysis was performed using StatSoft ${ }^{\circledR}$ statistical program STATISTICA 13.1. Distribution of variables was checked using the Shapiro-Wilk (W) test, while the homogeneity of variance was checked using the Fisher's test. The results were expressed as arithmetic means with standard deviations (SD). To compare the means, Student's t-test for independent variables was used. Tukey's test was used for post-hoc analysis. If no similarity of variance occurred, the non-parametric Kruskal-Wallis test was used. The study protocol was approved by the local Bioethics Committee (document no. KB 35/2020).

\section{Results}

The study group comprised 25 men and 20 women with the mean age of 62.34 years (men 61.24, women 64.56). In every analyzed CTA image, cerebral vessels were clearly visible and morphometric measurements were possible. Out of the 45 CTA images examined, 12 revealed unruptured MCA aneurysms, while 33 showed ruptured aneurysms and subarachnoid hemorrhage
(SAH). In 27 cases, the aneurysm was located on the right MCA, whereas in 18 cases on the left MCA. Due to the small sample size of the analyzed documentation, we decided not to compare the MCA morphometric parameters in patients with ruptured and unruptured aneurysms in this study. Since all CTAs concerned sporadic aneurysms in the population, the group was considered homogeneous.

A diagram of the measurements is shown in Figure 1. The number of $M 2$ branches originating in the $M 1$ division was also assessed. Mean values and standard deviations of the examined parameters of the MCA, as well as the angle between the MCA and ACA are presented in Table 1. The statistical analysis did not reveal any significant differences in terms of sex nor aneurysm laterality $(p>0.05)$ referring to both the occurrence of aneurysms and the parameters measured.

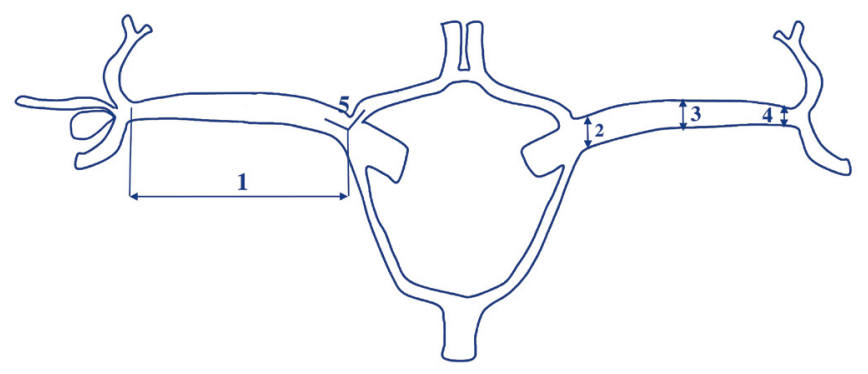

Figure 1. A diagram of the MCA measurements (1-5) and the number of $\mathrm{M} 2$ branches originating from the $\mathrm{M} 1$ division

Table 1. Values of the examined MCA parameters and ACA/MCA angle

\begin{tabular}{|c|c|c|c|c|c|c|c|}
\hline \multirow{2}{*}{$\begin{array}{c}\text { Measurement } \\
\text { parameters }\end{array}$} & \multirow{2}{*}{$\mathbf{N}$} & \multicolumn{2}{|c|}{ normal artery } & \multirow{2}{*}{$\mathbf{N}$} & \multicolumn{2}{|c|}{ artery with an aneurysm } & \multirow{2}{*}{$\mathbf{p}$} \\
\hline & & Mean & SD & & Mean & SD & \\
\hline length of M1 & 45 & 24.1 & 2.43 & 45 & 23.61 & 2.21 & 0.132 \\
\hline width of the proximal end & 45 & 2.19 & 0.19 & 45 & 2.21 & 0.17 & 0.142 \\
\hline width of the middle part & 45 & 1.99 & 0.12 & 45 & 2.02 & 0.14 & 0.234 \\
\hline width of the distal end & 45 & 1.88 & 0.15 & 45 & 1.92 & 0.13 & 0.145 \\
\hline angle of artery M1/A1 & 45 & 123.97 & 16.23 & 45 & 122.94 & 17.65 & 0.091 \\
\hline trifurcation & 17 & 4 & - & 17 & 12 & - & - \\
\hline
\end{tabular}


In the studied group, the mean length of segment $M 1$ was $24.1 \pm 2.43 \mathrm{~mm}$ on the non-affected side and $23.6 \pm 2.21 \mathrm{~mm}$ on the affected side. The mean width measured in the proximal end of the MCA was $2.19 \pm$ $0.19 \mathrm{~mm}$ on the non-affected side and $2.21 \pm 0.17 \mathrm{~mm}$ on the affected side. The mean width of the middle part of $\mathrm{M} 1$ was $1.99 \pm 0.12 \mathrm{~mm}$ on the non-affected side and $2.02 \pm 0.14 \mathrm{~mm}$ on the affected side. The mean widths measured next to the division initiating the $M 2$ segments on the non-affected and affected sides were: $1.88 \pm 0.15 \mathrm{~mm}$ and $1.92 \pm 0.13 \mathrm{~mm}$, respectively.

The angles of division between the ACA and MCA were: $123.97 \pm 16.23^{\circ}$ on the non-affected side and $122.94 \pm 17.65^{\circ}$ on the affected side. The CTAs visualized the types of the $\mathrm{M} 1 / \mathrm{M} 2$ division in terms of the number of branches. In the study group consisting of 90 arteries, a trifurcation was observed in 4 cases (8.8\%) of non-affected arteries and in 12 cases (26.6\%) of arteries with aneurysms. CTA with a MCA trifurcation with an aneurysm on the inferior side of the M1 division is shown in Figure 2.

\section{Discussion}

The MCA morphometric analysis of non-affected arteries and aneurysms is a subject of interest for numerous authors. However, the literature concentrates on the microsurgical anatomy of the MCA based on cadaver specimens. Valuable reports on the topic were published by Grand et al. (1980), Gibo et al. (1981), Umansky et al. (1984) and Yasargil (1984) $[1-3,11]$. Our study focused on CTA assessment of the $\mathrm{M} 1$ length and the number of $\mathrm{M} 2$ branches in living patients, not cadavers.

The mean length of $\mathrm{M} 1$ in our study was $24.1 \pm 2.43$ $\mathrm{mm}$ on the non-affected side and $23.6 \pm 2.21 \mathrm{~mm}$ on the affected side, which was greater than those reported by other authors. According to Umansky et al., it was 15.1 and $15.7 \mathrm{~mm}$ [11], respectively, and according to Yasargil, it was 14-16 mm [3]. On the basis of the microsurgical MCA anatomy in the Indian population, Pai et al. obtained the mean M1 length of $20.00 \mathrm{~mm}$ [6], which was relatively similar to our measurements [6]. In our study the M1 segment was bifurcated in 71 arteries (78.89\%) and trifurcated in 19 arteries (21.11\%). Comparable findings were published by Pai et al. [6], and earlier by Yasargil and Umansky et al. [3, 6, 11]. According to some authors, the M1 division is always a bifurcation and the presence of three or four main arterial branches results from a very early $\mathrm{M} 2$ division, which makes the $M 2$ segments originate in the $M 1$ division $[6,7,12]$. The traditional M1 division into two $\mathrm{M} 2$ branches (bifurcation) is observed in vascular studies in $50 \%$ of cases, even in approx. $80 \%$ according to some authors [6]. The two M2 branches are the superior and inferior ones.

According to the literature, in a bifurcation both branches may be equal in diameter or one of them may be dominant $[7,12]$. A trifurcation is observed in $12-25 \%$ of cases but according to some authors it is a pseudo-trifurcation with a very early division of

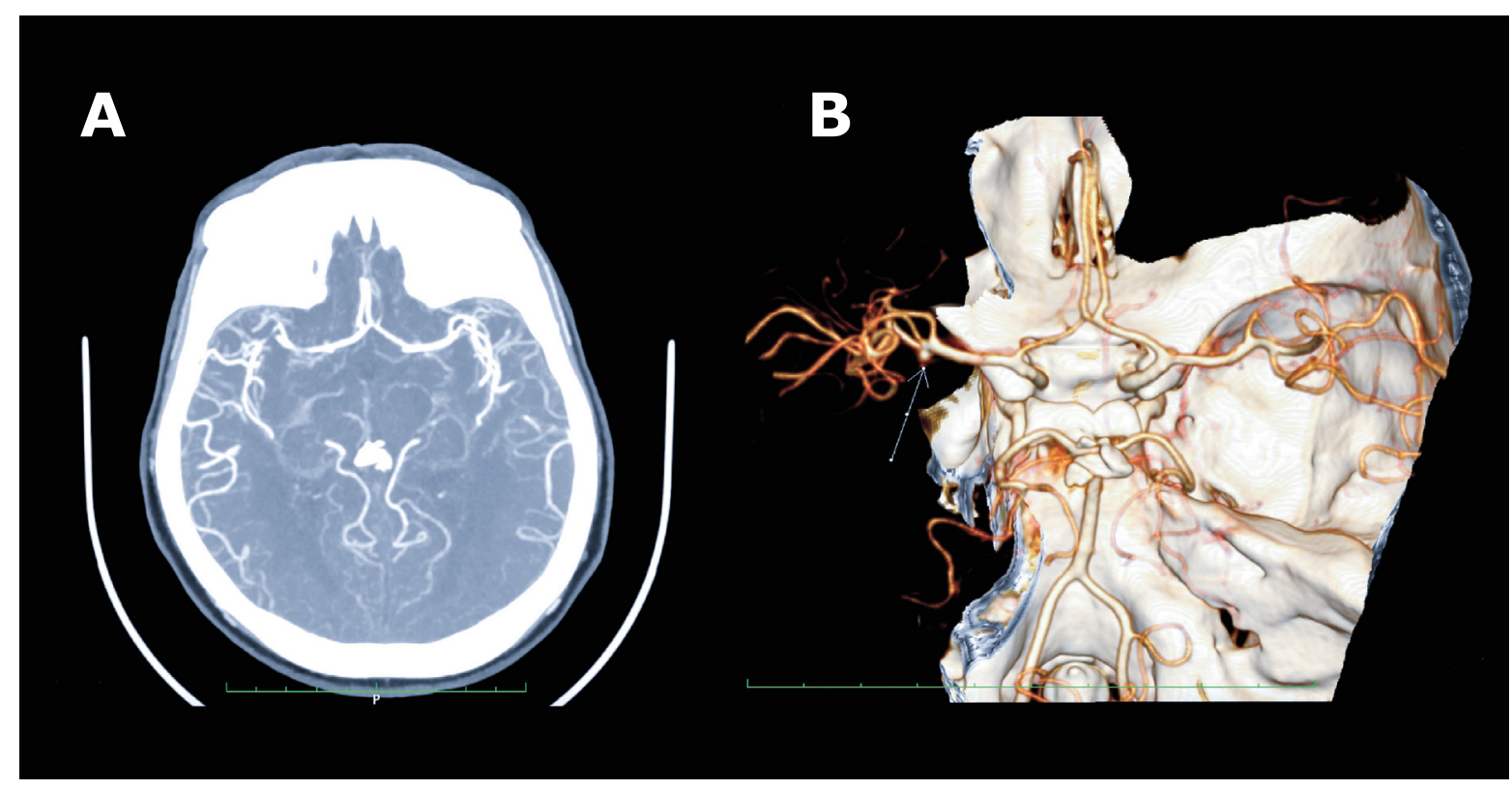

Figure 2. CTA two-dimensional axial projection with a M1 trifurcation with an aneurysm (A), CTA three-dimensional projection of the M1 division - a trifurcation of M1 with aneurysm (B) 
the $\mathrm{M} 2$ superior branch (in $15 \%$ of cases) or the $\mathrm{M} 2$ inferior branch (in $10 \%$ of cases). In $5 \%$ of cases, both branches are split early which gives the appearance of a pseudo-tetrafurcation. In $\sim 2 \%$ of cases, there is no apparent division of the $\mathrm{M} 1$ trunk into $\mathrm{M} 2$ branches, with one dominant $\mathrm{M} 2$ branch being the extension of $\mathrm{M} 1$ and the other smaller $\mathrm{M} 2$ branch appearing as a lateral branch $[6,12]$. In our study, the number of branches originating from the $\mathrm{M} 1$ division was counted. Out of 19 arteries with observed a MCA trifurcation, 15 were affected by an aneurysm, and only 4 were non-affected.

Some authors have pointed out that aneurysms of the MCA division are characterized by a wide neck covering the division. Thus, the anatomy of the MCA M1 division is distorted and the visible M1 trifurcation may in fact be an early division of one of the $\mathrm{M} 2$ branches, not a true trifurcation [11-12]. Yasargil claims that the exact anatomy of the MCA division with the number of branches can only be determined in traoperatively [3]. Orakdogen et al. published a DSA evaluation in 128 patients with aneurysms in the area of the circle of Willis, while our study is restricted to the CTA evaluation of MCAs in 45 patients with unilateral MCA aneurysms. Orkadogen et al. reported that in patients with MCA aneurysms, anatomical differences in cerebral arteries were much less common than in aneurysms of the anterior and posterior communicating arteries (ACOA and PCoA). Anatomical differences of the MCA in patients with aneurysms are more frequent in men. The most common anatomical variation was a trifurcation of the MCA [13]. In our study, no sex differences in the morphometric parameters of the MCA were found. As in our study, Orkadogen investigated ruptured aneurysms and those detected incidentally. Patients with incidentally detected aneurysms consituted $82.8 \%$, while those with ruptured aneurysms constituted $17.2 \%$ of the study group [13] In our study, the proportion was similar: $73.33 \%$ and $26.67 \%$, respectively. Both in our study and in that by Orkadogen et al., analyses of vascular examinations mostly included patients with SAH. Sadatomo et al. conducted an anatomical analysis of the MCA division in patients with aneurysms in that area and in participants with non-affected arteries.

Based on three-dimensional DSA and MRA, they evaluated the MCA division in non-affected arteries and in those with aneurysms of the M1 division, as in our study. The study group comprised 62 patients. They focused on the evaluation of the MCA division, measuring the division angles of $\mathrm{M} 2$ branches, their thickness and dominance of one branch [12]. We did not perform such measurements in this study. On the other hand, Sadatomo et al. excluded cases of a MCA trifurcation from their study. They also excluded cases, in which a ruptured aneurysm caused a intracerebral hematoma, which prevented evaluation of the MCA in imaging examination [12].

\section{Conclusions}

The morphometric parameters of the M1 segment of the MCA are not associated with the presence of an aneurysm in the $\mathrm{M} 1$ division. The coexistence of the more frequent trifurcation of the MCA and an aneurysm constitutes evidence of greater anatomical variability of this arterial area in cases with a concomitant vascular malformation.

\section{Acknowledgements}

The authors thank the computed tomography technicians and doctors of the Radiology Department for dedicating their skills and professionalism to the acquisition and analysis of images for this study.

\section{Declarations}

Ethics approval and consent to participate: The experiment was approved by the Bioethics Committee of the Ludwik Rydygier Collegium Medicum in Bydgoszcz (KB 35/2020). The study was a retrospective review of CTA images only without any personal data of patients used. In accordance with the approval of the Bioethics Committee, no written consent was required.

Consent for publication: The consent of the Bioethics Committee for the publication of this study was obtained.

Availability of data and materials: All relevant data is presented in the article.

Conflict of interest: The authors declare that they have no conflict of interest.

Funding: The study was financed from own funds of the Neurosurgery Department and the Department of Normal Anatomy of the Ludwik Rydygier Collegium Medicum in Bydgoszcz. The authors received no specific funding for this work. 


\section{References}

1. Grand W. Microsurgical Anatomy of the Proximal Middle Cerebral Artery and the Internal Carotid Artery Bifurcation. Neurosurgery [Internet]. 1980 Sep;7(3):215-8. Available from: https://academic.oup.com/neurosurgery/article-lookup/ doi/10.1227/00006123-198009000-00002

2. Gibo H, Carver CC, Rhoton AL, Lenkey C, Mitchell RJ. Microsurgical anatomy of the middle cerebral artery. J Neurosurg [Internet]. 1981 Feb;54(2):151-69. Available from: https://thejns.org/view/journals/j-neurosurg/54/2/article-p151.xml

3. Yaşargil MG. Microsurgical anatomy of the basal cisterns and vessels of the brain, diagnostic studies, general operative techniques and pathological considerations of the intracranial aneurysms. Microsurgery. 1984;25-143.

4. Liu Z, Cai Y, Guo-Zhong C, Lu G-M, Li Z-Y. Anatomical Variations in Circle of Willis and Intracranial Aneurysm Formation. Mol Cell Biomech [Internet]. 2017;14(1):19-31. Available from: https://search.proquest.com/scholarly-journals/anatomical-variations-circle-willis-intracranial/docview/2397225070/se-2?accountid=47980

5. Kaspera W, Ćmiel-Smorzyk K, Wolański W, Kawlewska E, Hebda A, Gzik M, et al. Morphological and Hemodynamic Risk Factors for Middle Cerebral Artery Aneurysm: a Case-Control Study of 190 Patients. Sci Rep [Internet]. 2020;10(1):2016. Available from: https://doi.org/10.1038/s41598-019-56061-2

6. Pai Sb, Varma R, Kulkarni R. Microsurgical anatomy of the middle cerebral artery. Neurol India [Internet]. 2005;53(2):186. Available from: http://www.neurologyindia.com/text.asp?2005/53/2/186/16406

7. Ring BA. Middle Cerebral Artery: Anatomical and Radiographic Study. Acta radiol [Internet]. 1962 Jul 1;Original S(4):289300. Available from: http://acr.sagepub.com/lookup/doi/10.1177/028418516205700407

8. Trivelato FP, Ulhôa AC, Abud DG, Rezende MTS. Intracranial aneurysm diameter and risk of rupture. Arq Neuropsiquiatr [Internet]. 2019 Nov;77(11):838-9. Available from: http://www.scielo.br/scielo.php?script=sci arttext\&pid=S0004-282X2019001100838\&tlng=en

9. Etminan N, Chang H-S, Hackenberg K, de Rooij NK, Vergouwen MDI, Rinkel GJE, et al. Worldwide Incidence of Aneurysmal Subarachnoid Hemorrhage According to Region, Time Period, Blood Pressure, and Smoking Prevalence in the Population. JAMA Neurol [Internet]. 2019 May 1;76(5):588. Available from: http://archneur.jamanetwork.com/article. aspx?doi=10.1001/jamaneurol.2019.0006

10. Feng Y, Shu SJ. Diagnostic Value of Low-Dose 256-Slice Spiral CT Angiography, MR Angiography, and 3D-DSA in Cerebral Aneurysms. Dis Markers [Internet]. 2020 Jan 13;2020:1-5. Available from: https://www.hindawi.com/journals/ $\mathrm{dm} / 2020 / 8536471 /$

11. Umansky F, Juarez SM, Dujovny M, Ausman JI, Diaz FG, Gomes F, et al. Microsurgical anatomy of the proximal segments of the middle cerebral artery. J Neurosurg [Internet]. 1984 Sep;61(3):458-67. Available from: https://thejns.org/view/ journals/i-neurosurg/61/3/article-p458.xml

12. Sadatomo T, Yuki K, Migita K, Imada Y, Kuwabara M, Kurisu K. Differences between middle cerebral artery bifurcations with normal anatomy and those with aneurysms. Neurosurg Rev [Internet]. 2013 Jul 26;36(3):437-45. Available from: http://link.springer.com/10.1007/s10143-013-0450-5

13. Orakdogen $\mathrm{M}$, Emon ST, Somay H, Engin T, Is M, Hakan T. Vascular variations associated with intracranial aneurysms. Turk Neurosurg [Internet]. 2017;27(6):853-62. Available from: http://www.turkishneurosurgery.org.tr/summary en doi. php3?doi=10.5137/1019-5149.JTN.17839-16.1 